\section{MS31-05 Self-organization of the reaction-fracture coupled front on the mesoscopic scale caused by morphological instability at $\mathrm{CuCl}_{2} \cdot 2 \mathrm{H}_{2} \mathrm{O}$ dehydration \\ Stanislav A. Chizhik ${ }^{1,2}$}

1. Novosibirsk State University; 630090 Pirogova, 2, Novosibirsk, Russia

2. Institute of Solid State Chemistry and Mechanochemistry, SB RAS; 630128 Kutateladze 18, Novosibirsk, Russia

email: csabox@yandex.ru

The kinetics of many heterogeneous solid state reactions can be described as propagation of the reaction-fracture coupled front appearing as a result of the feedback between the reaction and fracture [1].

Anisotropy of the mechanical properties of reagents results in that the rate of the reaction front depends on its crystallographic orientation. This can lead to a morphological instability of the front in some instances. If a reaction starts on a crystal face that corresponds to the orientation of slow movement then local perturbations of the front shape may result in formation of the front regions which move faster and so increasing initial disturbance. Initial flat front is partitioned to the pieces in which local crystallographic orientation differs from that of original front. On average the resulting reorganized front retains the initial front orientation but moves faster and produces different fracture morphology.

This picture was observed experimentally when studying dehydration of $\mathrm{CuCl}_{2} \square 2 \mathrm{H}_{2} \mathrm{O}$. The front starting from faces (110) moves in deep of about $1 \mathrm{mkm}$ retaining flat shape. Thereafter it partitions to sharp wedged pieces oriented as [010] and positioned in aperiodic manner distanced by $0.5-2 \mathrm{mkm}$ from each other (Fig. 1). Average rate of the front increases several times.

With the single-crystal X-ray diffraction it was found out that the product consists of nano-sized twin crystallites of two orientations. Deformation tensor computed from these relations is characterized by strong anisotropy. In two directions there is shrinkage by $12 \%$ and $37 \%$ whereas in third an expansion occurs by $6 \%$. We suggest that the product twins are formed in a ratio providing minimization of elastic strain energy. It is shown that the most significant decrease of the elastic strain energy can be achieved due to twining on the initial (110) crystal face. According to the model this results in significant slowing down of the reaction front in this direction. At the same time the twining can not provide a decrease of the stress for the front parallel to the face (100). Consequently such a front could be the fastest one. Thus the most likely perturbations of the initial flat front are the wedge-like formations directed along [010] which provide appearing of local front pieces slightly inclined to the fast direction (100). These theoretical results are fully confirmed by the experiments.

[1] S.A Chizhik, A.A. Sidelnikov, Solid State Ionics, 178 (2007), 1487

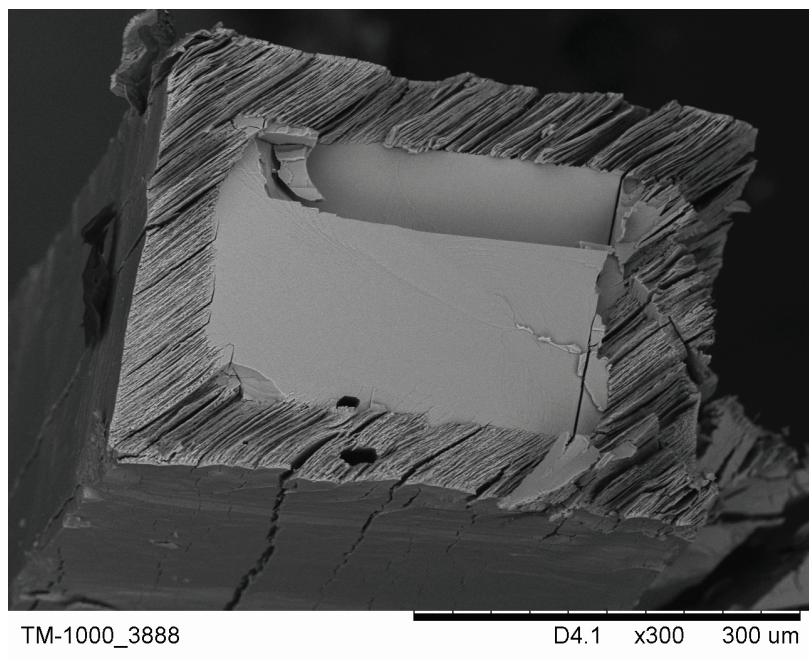

Figure 1. The crystal cut demonstrating the reaction-fracture front propagating from the faces (110)

Keywords: solid state reactions, fracture, feedback, morphology, self-organization 